\title{
DINÂMICA DOS ATRIBUTOS FÍSICO-QUÍMICOS E VARIAÇÃO SAZONAL DOS ESTOQUES DE CARBONO NO SOLO EM DIFERENTES FITOFISIONOMIAS DO PANTANAL NORTE MATO-GROSSENSE ${ }^{1}$
}

\author{
Juliana Milesi Mello², Eduardo Guimarães Couto ${ }^{3}$, Ricardo Silva Santos Amorim³, Léo Adriano Chig ${ }^{4}$, \\ Mark Stephen Johnson ${ }^{5}$ e Francisco Almeida Lobo ${ }^{3}$
}

\begin{abstract}
RESUMO - O bioma Pantanal pode ser considerado um ambiente de hipersazonalidade, onde há um período de inundação pronunciada seguido por extenso período seco com temperaturas muito elevadas. A diversidade de suas fitofisionomias ocasiona aportes de carbono para o solo em quantidade e qualidade distintas. Com esse foco, este trabalho teve por objetivo verificar o efeito do ciclo hidrológico do Pantanal (enchente, cheia, vazante e estiagem) sobre a dinâmica do estoque carbono orgânico do solo nas fitofisionomias de Cambarazal, de Floresta Ripária, de Cordilheira e de Espinheiral. Verificou-se que as fitofisionomias puderam ser separadas pela análise de componentes principais (ACP) em três grupos distintos, caracterizados pela reserva de nutrientes e pela acidez do solo. O estoque de carbono do solo está condicionado por fatores sazonais e igualmente dependentes da fitofisionomia local que ocorreram nas fitofisionomias que apresentaram maior nível de fertilidade natural, ou seja, respectivamente o Cambarazal e o Espinheiral. O maior teor de carbono foi observado no período de enchente, na maioria das fitofisionomias, e o menor estoque de carbono aconteceu no período de vazante (2008), após o período de anaerobiose em razão da enchente e antes que o período seco passasse a ser determinante.
\end{abstract}

Palavras-chave: Gleissolos; Planossolos; Solos Hidromórficos

\section{DYNAMICS OF CHEMICAL AND PHYSICAL ATTRIBUTES AND SEASONAL VARIATION OF SOIL CARBON STOCKS IN DIFFERENT VEGETATION TYPES OF NORTH PANTANAL OF MATO GROSSO}

\begin{abstract}
The Pantanal biome may be considered a hyperseasonal environment where there is a flooded period followed by an extended dry period with very high temperatures. The diversity of vegetation types modify the input of carbon into the soil in different quantity and quality. With this focus, this study aimed to verify the effect of the hydrological Pantanal Cycle (rising, flood, low tide and dry season), on the dynamics of soil organic carbon stock in different vegetation types of Cambarazal, Riparian Forest, Mountain Range and Thorn-Scrub. Principal component analysis (PCA) identified three distinct groups, characterized by nutrient reserves and by soil acidity. The carbon stock of the soil is conditioned by seasonal factors and equally dependent on the local vegetation type that occurred in vegetation types that showed the highest level of natural fertility, respectively, Cambarazal and Thorn-Scrub. The highest carbon content was observed during the flood period, for most vegetation types and the lowest carbon stock occurred during the period of low tide in 2008, just after a period of anaerobiosis due to the flood and before the dry season became predominant.
\end{abstract}

Keywords: Gleyssols; Planosols; Hydromorphic Soils

\footnotetext{
${ }^{1}$ Recebido em 29.04.2013 aceito para publicação em 15.12.2014.

${ }^{2}$ Universidade Federal de Mato Grosso, UFMT, Mestrado em Agricultura Tropical, Cuiabá, MT - Brasil. E-mail: $<$ jumilesi@hotmail.com>.

${ }_{3}^{3}$ Universidade Federal de Mato Grosso, UFMT, Faculdade de Agronomia, Medicina Veterinária e Zootecnia, Departamento de Solos e Engenharia Rural, Cuiabá, MT - Brasil.E-mail: <couto@ufmt.br $>$, < rsamorim@ufmt.br $>$ e $<$ fdealobo@gmail.com>. ${ }^{4}$ Universidade de Cuiabá, UNIC, Cuiabá, MT - Brasil. E-mail: <leochig@gmail.com>.

${ }^{5}$ University of British Columbia, Institute for Resources, Environment and Sustainability, Vancouver - Canadá. E-mail: <mark.johnson@ubc.ca>.
} 


\section{INTRODUÇ̃̃O}

O balanço global de carbono do ecossistema só pode ser inteiramente dimensionado se a contribuição de cada processo envolvido na emissão e consumo do carbono for levada em consideração. O estoque de carbono nos solos, por exemplo, em escala global, excede na proporção de 4:1 àquele encontrado na vegetação e, em particular, as áreas alagadas que correspondem a 5\% de toda a área da superfície terrestre contêm aproximadamente $40 \%$ de todo o carbono orgânico do solo (COS). A avaliação da dinâmica do estoque de carbono nos solos dessas áreas alagadas assume relevante importância no contexto das mudanças climáticas globais, especificamente no efeito-estufa, devido à potencial contribuição do $\mathrm{CO}_{2}$ proveniente do solo (SMITH et al., 2008; JOHNSTON et al., 2009; EAGLE; OLANDER, 2012; JOHNSON et al., 2013).

A quantidade de COS é altamente variável e influenciada por fatores de formação do solo como o clima, biota, topografia e material originário operando todos em conjunto. Nas áreas alagadas, a matéria orgânica é substrato dos microrganismos anaeróbicos, contribuindo para a redução da pressão parcial de $\mathrm{O}_{2}$ e sendo a causa de alterações específicas do potencial redox, do $\mathrm{pH}$ e da disponibilidade de nutrientes (NEUE, 1985). Além disso, o acúmulo de matéria orgânica do solo para níveis capazes de proporcionar suprimento adequado de água e nutrientes para a biota representa uma etapa fundamental no desenvolvimento inicial dos ecossistemas (BECHTOLD; NAIMAN, 2009).

Nas áreas alagadas, a cinética do processo de redução e os produtos gerados por esse processo são afetados pela quantidade e qualidade da matéria orgânica do solo (NEUE, 1985). Em geral, nas áreas permanentemente alagadas, como são as turfas, a taxa de decomposição da matéria orgânica tende a ser menor do que nas áreas não inundadas, promovendo, nesses casos, o acúmulo de carbono no solo (HOLDEN, 2005). No entanto, sabe-se que ciclos consecutivos de seca e umedecimento dos solos causam incremento nas taxas de mineralização da matéria orgânica e, consequentemente, de perda de nutrientes como nitrogênio e carbono, fenômeno descrito como efeito Birch (BIRCH, 1964). Além disso, o COS é um determinante importante das funções ecológicas das áreas úmidas. Por exemplo, a redução do nitrato e a transformação dos nutrientes orgânicos em formas disponíveis para o crescimento das plantas são reguladas pelos nutrientes e energia contidos no reservatório de carbono do solo (BRADY; WEIL, 1999).

No pantanal do norte mato-grossense existem diferentes fitofisionomias como são as matas, os cerradões e as savanas (Cerrado), sendo a maior parte, aproximadamente $58 \%$, de florestas inundáveis (IBGE, 1992; SILVA et al., 2000), além de campos inundáveis como brejos, lagoas e vazantes. Essas fitofisionomias distintas são responsáveis por prover ao solo matéria orgânica com características quantitativas e qualitativas visivelmente diferentes. Além disso, esse ambiente experimenta anualmente momentos extremos de excesso no período de inundação e de falta de umidade no solo no período seco. Ao longo dessas mudanças anuais ocorrem alterações tanto na paisagem natural quanto nos atributos químicos do solo, por existirem águas paradas e correntes, temporárias ou permanentes, sobre solos predominantemente profundos, pouco estratificados, lixiviados e pobres em resíduos orgânicos. Assim, é plausível esperar que, nesse ecossistema, a dinâmica do estoque de carbono e da composição físico-química do solo seja condicionada tanto pela composição de espécies vegetais quanto pela sazonalidade ambiental, com ciclos de seca e de inundação.

O conhecimento da dinâmica do carbono em áreas alagáveis tem sido levantado por pesquisas, em sua maioria, direcionadas para subsidiar o manejo adequado do solo para práticas agrícolas, como o cultivo de arroz irrigado por alagamento (GUO et al., 2012). Assim como em outros ecossistemas naturais brasileiros, o entendimento sobre a dinâmica do carbono no solo do Pantanal ainda está sendo construído.

Por tal razão, este trabalho teve como objetivos identificar fitofisionomias com atributos físico-químicos comuns e avaliar as variações sazonais nos estoques de carbono no solo para os grupos fitofisionômicos seguirem um padrão igualmente comum.

\section{MATERIAL E MÉTODOS}

\section{1. Área de estudo, fitofisionomias e períodos sazonais}

A Reserva Particular de Patrimônio Natural do Serviço Social do Comércio (RPPN SESC Pantanal) está localizada no Município de Barão de Melgaço, Estado de Mato Grosso (Figura 1AB). A RPPN possui uma

Revista Árvore, Viçosa-MG, v.39, n.2, p.325-336, 2015

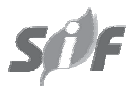


área de 106.300 ha, localizando-se no interflúvio dos rios Cuiabá e São Lourenço.

As temperaturas médias na RPPN variam no verão entre $27^{\circ}$ e $28^{\circ} \mathrm{C}$ e durante o inverno entre $22^{\circ}$ e $23^{\circ} \mathrm{C}$, com a precipitação oscilando entre 1.100 e $1.200 \mathrm{~mm}$. Desses, entre 450 e $525 \mathrm{~mm}$ estão concentrados no trimestre janeiro, fevereiro e março (HASENACK et al., 2003) e um período seco prolongado que vai de maio a setembro. A topografia é plana, causando alagamento excessivo durante o período úmido que pode alcançar até 2,0 m (NUNES DA CUNHA; JUNK, 2001). As oscilações climáticas têm-se mostrado a principal causa dos ciclos plurianuais de inundação (JUNK; STRUSSMANN, 2006).

O estudo foi realizado em quatro fitofisionomias: Cambarazal: Fitofisionomia caracterizada pela presença dominante do cambará [Vochysia divergens Pohl (Vochysiaceae)]. Consiste numa área densamente

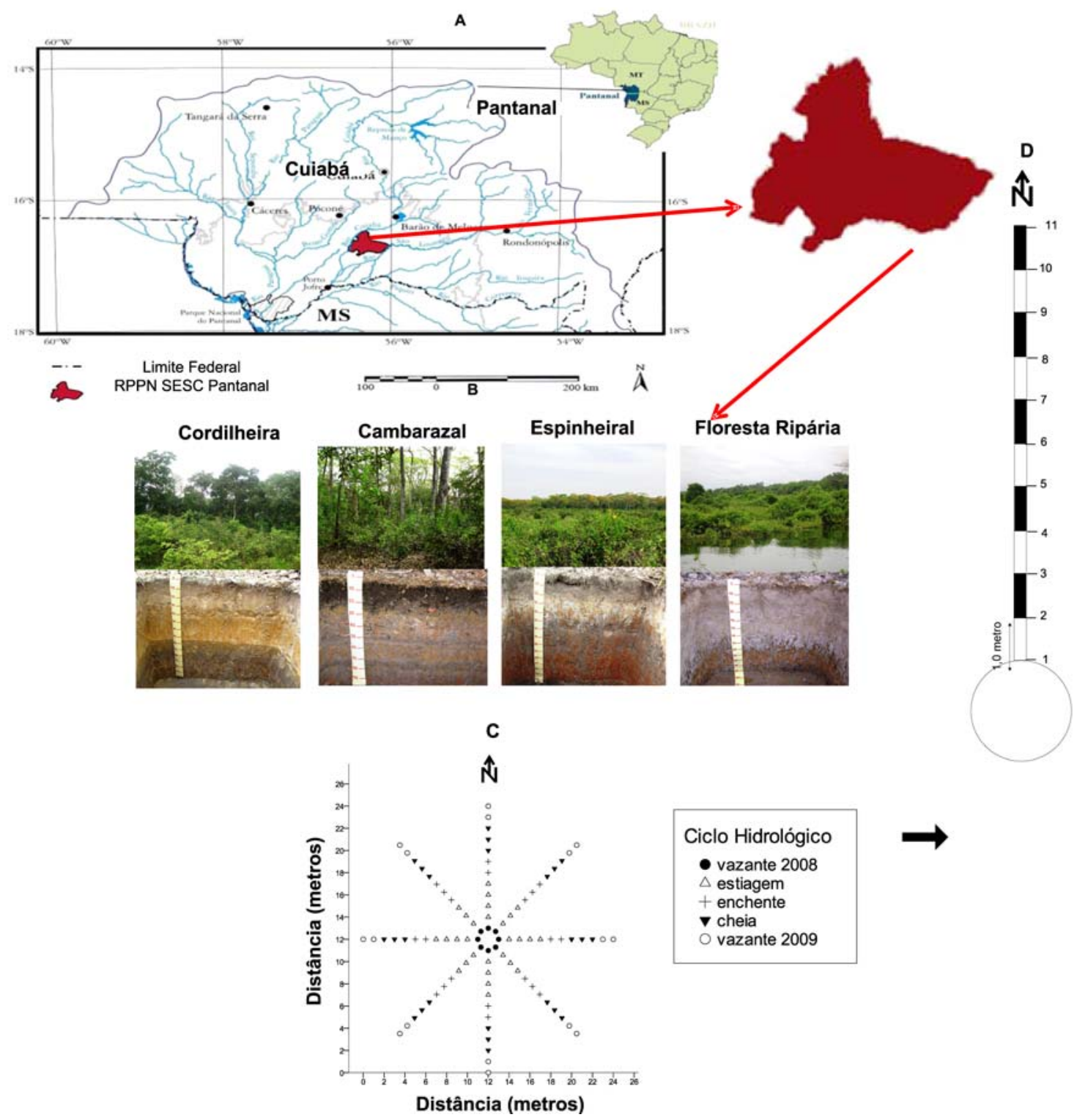

Figura 1 - Localização da área, esquema de amostragem e detalhes sobre as fitofisionomias e perfis de solos. Figure 1 - Location of the area, sampling scheme and details of the vegetation types and soil profiles. 
florestada e numa área alagada sazonalmente. Floresta Ripária: Fisionomia florestal, onde o estrato arbóreo emergente é esparso e o sub-bosque, pouco desenvolvido. Inclui fisionomia "Mista" e equivale aos "Cerrados" e "Cerradões". Cordilheira: Fitofisionomia associada às pequenas elevações em forma de cordões que ocorrem na paisagem e são caracterizadas pela presença de Savana arbórea densa com predomínio da lixeira [Curatella americana L. (Dilleniaceae)], do cumbaru [Dipteryx alata Vog. (Leguminosae-Papilionoideae)] e com o sub-bosque marcado pela presença de acuri [Scheelea phalerata (Mart.) Burret (Palmae)]. Espinheiral: Fitofisionomia com predominância de vegetação arbustiva caracterizada pela espécie Mimosa pellita H. et B. (LeguminosaeMimosoideae) denominada localmente espinheiral e gramíneas, como a taquarinha [Gymnopogon spicatus (Spreng.) Kuntze (Gramineae-Poaceae)]. As áreas de estudo compreenderam pequenos transectos que tiveram a finalidade de caracterizar a variabilidade das fitofisionomias a curta distância, conforme apresentado na Figura 1. A análise dos dados de granulometria dos solos no triângulo textural variou entre areia-franca e argila (Figura 2).

Nos locais de coletas foram abertas trincheiras para identificação e classificação dos solos de acordo com os procedimentos descritos por Lemos et al. (2005), considerando-se a grande diversidade de espécies vegetativas que pode ocorrer em virtude das variações na textura e da fertilidade do solo (RODRIGUES et al., 2007). As amostras foram coletadas de acordo com o esquema de amostragem mostrado na Figura 1CD. Para a caracterização dos solos, foram coletadas 11 amostras espaçadas de 1,0 m e dispostas em um pequeno transecto, na direção N-S.

As coletas das amostras de solo foram realizadas com o auxílio de anel volumétrico com volume de 50 $\mathrm{cm}^{3}$, para o estudo do COS e de seu estoque. São realizadas em círculos concêntricos (Figura 1C), nas profundidades de 0 a 5,5 a 10,10 a 15 e 15 a $20 \mathrm{~cm}$, com raios crescentes de $1,0 \mathrm{~m}$ a cada 15 dias (coletados no período de abril de 2008 a julho de 2009), perfazendo um total de 1.536 amostras após as 24 coletas.

Com essas coletas, foi possível avaliar todos os períodos sazonais da hidrologia característicos da região, que são de enchente, de cheia, de vazante e de estiagem. Além disso, especificamente no período de vazante, foi possível realizar medições em 2008 e em 2009.

Revista Árvore, Viçosa-MG, v.39, n.2, p.325-336, 2015

\subsection{Atributos físico-químicos dos solos}

A avaliação dos atributos físico-químicos dos solos foi realizada apenas no período de vazante de 2008 . Os atributos físicos e químicos dos solos se prestaram para identificar a possibilidade de agrupar as fitofisionomias com características comuns, como mencionado no item "análises estatísticas".

A caracterização físico-química dos solos foi baseada em 16 indicadores: $\mathrm{pH} \mathrm{H}_{2} \mathrm{O}$, potássio $(\mathrm{K})$ e fósforo $(\mathrm{P})$ (Mehlich 1), cálcio (Ca), magnésio ( $\mathrm{Mg})$, alumínio (Al), carbono orgânico $(\mathrm{CO})$, zinco $(\mathrm{Zn})$, cobre $(\mathrm{Cu})$, ferro $(\mathrm{Fe})$, manganês $(\mathrm{Mn})$, enxofre (S), boro (B), argila e densidade do solo.

$\mathrm{O}$ pH em $\mathrm{H}_{2} \mathrm{O}$ foi determinado potenciometricamente em suspensão solo/líquido, na proporção 1:2,5, com tempo de repouso de uma hora e agitação antes da leitura.

O fósforo, potássio, sódio, cobre, ferro e zinco foram extraídos com solução Mehlich $1(\mathrm{HCl}$ 0,05 M $+\mathrm{H}_{2} \mathrm{SO}_{4} 0,0125 \mathrm{M}$ ), sendo o fósforo determinado por

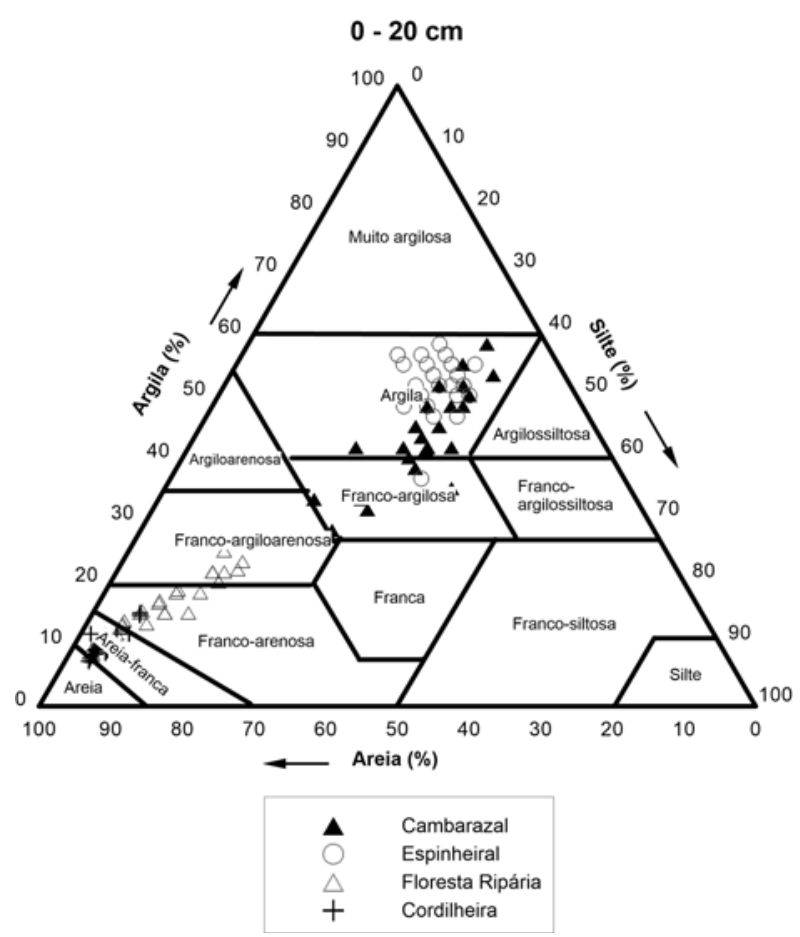

Figura 2-Classes texturais das amostras de solo na profundidade de áreas estudadas.

Figure 2 - Textural classes of soil samples at a depth of study areas. 
fotocolorimetria (molibdato de amônio e ácido ascórbico), o potássio e o sódio por fotometria de chama e o cobre, o ferro e o zinco quantificados por meio de espectrometria de absorção atômica, em que os limites de detecção (LD) e de quantificação (LQ) foram calculados a partir do desvio-padrão do branco analítico e do coeficiente angular da curva de calibração de cada elemento (LONG; WINEFORDNER, 1983).

O B foi determinado pela água quente, com filtragem após a decantação de $16 \mathrm{~h}$, e quantificado por meio de espectrometria de absorção atômica; o manganês, por espectrofotometria de absorção atômica, enquanto o alumínio, cálcio e magnésio, por titulação com $\mathrm{NaOH} 0,025 \mathrm{~N}$ e EDTA $0,025 \mathrm{~N}$.

Os teores de carbono do solo foram determinados nas amostras coletadas ao longo do ciclo hidrológico do pantanal mato-grossense pelo do Método de Combustão Seca, utilizando-se o analisador Multi NC 3.000 (Analytik Jena, Germany) e forno Eltra, cuja temperatura se eleva até $1.350^{\circ} \mathrm{C}$, conforme metodologia descrita pelo Soil Survey Staff (1996). Para transformar os teores de carbono em valores de estoque de carbono no solo obtidos em todos os períodos sazonais da hidrologia característicos da região, empregou-se a equação 1 .

$$
\text { Cest }=C \times P \times \text { Dap } / 10
$$

em que Cest $=$ estoque de carbono no solo à profundidade $\mathrm{P}$ considerada $\left(\mathrm{t} \mathrm{ha}^{-1}\right) ; \mathrm{C}=$ teor de carbono $\left(\mathrm{g} \mathrm{kg}^{-1}\right)$; $\mathrm{P}=$ profundidade na qual a amostra de solo, para quantificar o teor de carbono e a densidade aparente, foi coletada $(\mathrm{cm})$; e Dap = densidade aparente do solo no intervalo de profundidade considerado $\left(\mathrm{g} \mathrm{dm}^{-3}\right)$.

Os teores de $\mathrm{H}+\mathrm{Al}$ (acidez total) foram obtidos com extrator acetato de cálcio em pH 7,0, que foram dosados por titulometria com EDTA.

Para a classificação textural, foi feito o uso do triângulo textural (SANTOS et al., 2005), utilizando-se a granulometria, que foi determinada pelo método do densímetro de Bouyoucos (KIEHL, 1979), após a dispersão com $\mathrm{NaOH} 0,1 \mathrm{~mol} \mathrm{~L}^{-1}$. A densidade aparente (Dap) foi determinada por meio de amostras de solo com estrutura indeformada, coletadas com anéis de aço de Kopecky de bordas cortantes com volume interno de $50 \mathrm{~cm}^{3}$ e secas na estufa a $105^{\circ} \mathrm{C}$, por 24 a 48 h.



\subsection{Análise estatística dos dados}

As análises estatísticas foram realizadas em duas etapas. A primeira consistiu no emprego da análise das componentes principais (PCA) para analisar a estrutura das inter-relações (correlações) entre o grande número de variáveis provenientes das análises físicoquímicas dos solos, coletadas na profundidade de 0 a $20 \mathrm{~cm}$, definindo um conjunto de dimensões latentes comuns que facilitam a compreensão da estrutura da nuvem de dados, chamados de fatores. Com o emprego dessa técnica, inicialmente se podem identificar as dimensões isoladas da estrutura dos dados e, então, determinar o grau em que cada variável é explicada por cada dimensão ou fator. A estrutura inicial utilizada para determinar a matriz de cargas fatoriais, em geral, pode não fornecer um padrão significativo de cargas das variáveis, por isso não é definitiva. A confirmação ou não dessa estrutura inicial pode ser feita por meio de vários métodos de rotação dos fatores (DILLON; GOLLDSTEIN, 1984; REIS, 2001). Nesta pesquisa, utilizou-se o método Varimax de rotação ortogonal dos fatores. O Varimax é um processo em que os eixos de referência dos fatores são rotacionados em torno da origem até que alguma outra posição seja alcançada. O objetivo foi redistribuir a variância dos primeiros fatores para os demais e atingir um padrão fatorial mais simples e teoricamente mais significativo (REIS, 2001).

A escolha dos fatores foi realizada por meio da técnica de raiz latente, conforme Hair JR. et al. (2005). A matriz de cargas fatoriais, que mede a correlação entre os fatores comuns e as variáveis observáveis, foi determinada por meio da matriz de correlação, conforme Jolliffe (2002). Essas análises foram realizadas no software SPSS 19 (SPSS, 2010).

A segunda etapa do estudo foi centrada apenas nos valores do estoque de carbono dos solos na profundidade de 0 a $20 \mathrm{~cm}$, obtidos pela soma dos estoques nas profundidades de 0 a 5,5 a 10,10 a 15 e 15 a $20 \mathrm{~cm}$. Em razão da ausência de normalidade dos dados mesmo após o emprego das transformações usuais, optou-se pelo uso da estimativa da média empregando a técnica de "bootstrap", com 1.000 reamostragens aleatórias com reposição, conforme a metodologia descrita por Christie (2004). A partir desse universo de 1.000 valores, também foi possível estabelecer os limites superior e inferior do intervalo de confiança da média, a 95\% de probabilidade, sendo esse

Revista Árvore, Viçosa-MG, v.39, n.2, p.325-336, 2015 
procedimento útil para a posterior comparação das médias entre si. Assim, médias com valores comuns dentro de seus intervalos de confiança não diferiram entre si, ao passo que a ausência de valores comuns indicou diferença significativa (a 5\% de probabilidade) entre elas. Essas análises permitiram verificar se as fitofisionomias agrupadas na primeira etapa do trabalho apresentariam ou não comportamento igualmente semelhante do ponto de vista do estoque de carbono no solo.

\section{RESULTADOS}

A partir das análises realizadas para a vazante de 2008, foi possível discriminar, pela análise de PCA, três grupos distintos do ponto de vista físico-químico do solo, considerando-se os dois primeiros PCAs. Esses componentes retêm $78 \%$ das informações e estão classificados em ordem decrescente de magnitude, como pode ser observado nas setas que expressam o tamanho dos erros da Figura $3 \mathrm{~A}$.

O primeiro componente pode ser caracterizado pela reserva de nutrientes (fases minerais e orgânicas) e o segundo componente, pela acidez do solo. O primeiro componente permitiu separar o conjunto de variáveis em três grupos (Figura 3A). Um grupo formado pelo Cambarazal, caracterizado pela presença de Gleissolos de textura argilosa (Figura 2) e que apresentou teores de matéria orgânica e reserva de nutrientes mais elevados (Tabela 1), seguido pelo grupo formado pelo Espinheiral, que apresentou valores intermediários, e pelo grupo formado pela Cordilheira e Floresta Ripária, que não se distinguiram uma da outra e exibiram os menores valores dessas variáveis.

O primeiro grupo (Cambarazal) apresentou valor médio do primeiro componente significativamente mais alto $(\mathrm{p}<0,001)$ do que o dos outros grupos (Figura 3B). O segundo grupo (Espinheiral), também caracterizado pela presença de Gleissolos argilosos, apresentou valor médio do primeiro componente significativamente mais alto $(\mathrm{p}<0,001)$ do que o do terceiro grupo (Floresta Ripária e Cordilheira). Esse grupo é caracterizado pela presença de Planossolos com horizonte A, de textura superficial arenosa (Figura 2) e com grande limitação, no que se refere à disponibilidade de nutrientes.

O segundo componente também permitiu distinguir três grupos. Um grupo formado pelo Espinheiral, que apresentou a menor acidez, expressa pelos menores valores de alumínio trocável $\left(\mathrm{Al}^{3+}\right)$; e os valores elevados de Fe, Ca e Mg. O segundo grupo, formado pela Floresta Ripária e Cordilheira, que não se distinguiram entre si, apresentou os valores intermediários dessas variáveis. O terceiro grupo é formado pelo Cambarazal, que mostrou os valores mais elevados de $\mathrm{Al}^{3+}$ e os mais baixos de Ca e Mg (Figura 3A).

O primeiro grupo (Espinheiral) apresentou valor médio do segundo componente significativamente mais alto ( $\mathrm{p}<0,001)$ do que o dos outros grupos (Figura 3B). O segundo grupo (Floresta Ripária e Cordilheira) exibiu
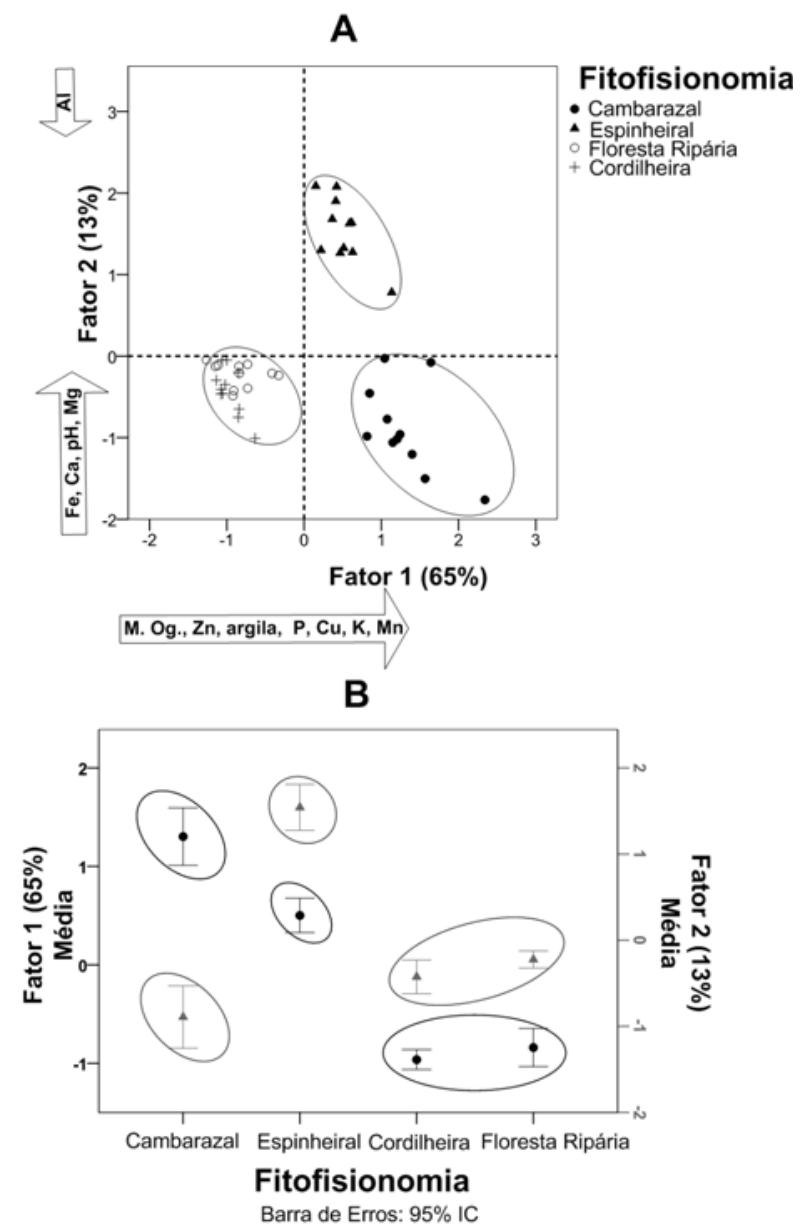

Figura 3 - Escores totais (A) e escores médios (B) dos fatores nos componentes principais 1 e 2 dos atributos químicos e físicos dos solos, coletados na vazante de 2008 (Barra de erros $=95 \%$ I.C.).

Figure 3 - Total scores (A) and mean scores (B) of the factors in principal components 1 and 2 of the chemical and physical properties of soils collected at low tide, 2008 (Error bar $=95 \%$ CI). 
Tabela 1 - Estatística descritiva dos parâmetros avaliados nas amostras de solos. Table 1 - Summary statistics from evaluated parameters in soil samples.

\begin{tabular}{|c|c|c|c|c|c|c|c|c|c|c|c|c|c|c|c|c|c|}
\hline & & $\mathrm{H} \mathrm{H}_{2} \mathrm{O}$ & $\mathrm{P}$ & $\mathrm{K}$ & $\mathrm{Ca}$ & $\mathrm{Mg}$ & $\mathrm{Al}$ & Argila & Silte & Areia & M.O. & $\mathrm{Zn}$ & $\mathrm{Cu}$ & $\mathrm{Fe}$ & Mn & S & B \\
\hline & & & - $(\mathrm{mg}$ & $\left(m^{-3}\right)-$ & & $\mathrm{mol}_{\mathrm{c}} \mathrm{d}$ & - & 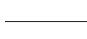 & $-(\mathrm{g} \mathrm{k}$ & $\left.g^{-1}\right)$ & - & & & $-(\mathrm{mg} \mathrm{d}$ & $\left(m^{-3}\right)$ & & \\
\hline \multirow[t]{5}{*}{ Cambarazal } & Mínimo & 4,1 & 1 & 40 & 0,2 & 0,2 & 0,3 & 284 & 217 & 83 & 19 & 1,3 & 0,9 & 90,0 & 13,0 & 4,8 & 0,2 \\
\hline & Máximo & 5,4 & 317 & 120 & 3,5 & 1,4 & 3,1 & 584 & 400 & 449 & 60 & 16,4 & 2,6 & 427,0 & 250,0 & 31,5 & 0,6 \\
\hline & Amplitude & 1,3 & 315 & 80 & 3,3 & 1,2 & 2,8 & 300 & 183 & 366 & 41 & 15,1 & 1,7 & 337,0 & 237,0 & 26,7 & 0,4 \\
\hline & Média & 4,8 & 56 & 76 & 0,9 & 0,6 & 1,4 & 439 & 319 & 242 & 41 & 4,8 & 1,6 & 231,7 & 50,4 & 12,7 & 0,3 \\
\hline & \multicolumn{2}{|c|}{ Desvio-padrão0,3 } & 91 & 20 & 0,8 & 0,3 & 1,0 & 73 & 36 & 91 & 11 & 3,5 & 0,5 & 76,1 & 44,3 & 6,3 & 0 \\
\hline & $\mathrm{CV} \%$ & 7 & 162 & 26 & 82 & 54 & 69 & 17 & 11 & 38 & 26 & 72 & 29 & 33 & 88 & 49 & 27 \\
\hline \multirow[t]{5}{*}{ Cordilheira } & Mínimo & 4,5 & 2 & 28 & 0,1 & 0,1 & 0,3 & 73 & 14 & 783 & 3 & 0,3 & 0,3 & 175,0 & 1,0 & 2,7 & 0,2 \\
\hline & Máximo & 5,3 & 5 & 53 & 1,0 & 0,6 & 1,0 & 151 & 67 & 894 & 27 & 2,0 & 0,8 & 372,0 & 43,6 & 9,9 & 0,5 \\
\hline & Amplitude & 0,8 & 3 & 25 & 0,9 & 0,5 & 0,7 & 78 & 53 & 111 & 24 & 1,7 & 0,5 & 197,0 & 42,6 & 7,2 & 0,3 \\
\hline & Média & 4,8 & 4 & 39 & 0,5 & 0,3 & 0,6 & 97 & 41 & 862 & 13 & 0,8 & 0,5 & 248,8 & 16,0 & 5,4 & 0,3 \\
\hline & \multicolumn{2}{|c|}{ Desvio-padrão0,2 } & 1 & 6 & 0,3 & 0,1 & 0,1 & 22 & 14 & 33 & 7 & 0,4 & 0,1 & 51,7 & 11,9 & 2,1 & 0 , \\
\hline \multicolumn{18}{|c|}{  } \\
\hline & $\mathrm{CV} \%$ & 4,2 & 23 & 16 & 52 & 53 & 23 & 22 & 33 & 4 & 55 & 47 & 25 & 21 & 75 & 40 & 35 \\
\hline \multirow[t]{6}{*}{ Espinheiral } & Mínimo & 4,3 & 1 & 19 & 1,3 & 0,5 & 0,0 & 367 & 217 & 116 & 5 & 1,1 & 0,9 & 216,0 & 15,9 & 6,8 & 0,1 \\
\hline & Máximo & 5,9 & 55 & 147 & 4,8 & 1,8 & 3,8 & 584 & 350 & 283 & 53 & 12,2 & 2,3 & 471,0 & 122,7 & 25,7 & 0,4 \\
\hline & Amplitude & 1,6 & 54 & 128 & 3,5 & 1,3 & 3,8 & 217 & 133 & 167 & 48 & 11,1 & 1,4 & 255,0 & 106,8 & 18,9 & 0,3 \\
\hline & Média & 4,9 & 20 & 77 & 2,7 & 1,0 & 1,7 & 524 & 298 & 178 & 28 & 4,7 & 1,5 & 408,4 & 53,0 & 14,4 & 0,2 \\
\hline & Desvio-padr & ão0,6 & 18 & 45 & 1,0 & 0,4 & 1,4 & 42 & 37 & 37 & 17 & 3,0 & 0,3 & 56,1 & 27,4 & 4,9 & 0,1 \\
\hline & $\mathrm{CV} \%$ & 13 & 94 & 58 & 38 & 41 & 86 & 8 & 13 & 21 & 60 & 64 & 23 & 14 & 52 & 34 & 27 \\
\hline \multirow[t]{6}{*}{ Floresta Riparia } & Mínimo & 4,5 & 1 & 24 & 0,2 & 0,2 & 0,4 & 81 & 33 & 599 & 4 & 0,2 & 0,5 & 175,0 & 1,0 & 2,3 & 0,1 \\
\hline & Máximo & 5,3 & 48 & 53 & 1,3 & 0,7 & 1,8 & 251 & 167 & 886 & 24 & 2,1 & 1,7 & 417,0 & 27,4 & 8,9 & 0,4 \\
\hline & Amplitude & 0,8 & 47 & 29 & 1,1 & 0,5 & 1,4 & 170 & 134 & 287 & 20 & 1,9 & 1,2 & 242,0 & 26,4 & 6,6 & 0,2 \\
\hline & Média & 4,7 & 8 & 39 & 0,6 & 0,4 & 1,1 & 170 & 99 & 731 & 11 & 0,7 & 0,8 & 244,9 & 7,3 & 4,4 & 0,2 \\
\hline & Desvio-padrão & 0,2 & 11 & 8 & 0,3 & 0,1 & 0,5 & 43 & 42 & 82 & 5 & 0,5 & 0,3 & 46,1 & 7,0 & 1,7 & 0,0 \\
\hline & $\mathrm{CV} \%$ & 5 & 152 & 20 & 58 & 28 & 45 & 25 & 42 & 11 & 43 & 63 & 33 & 19 & 96 & 38 & 24 \\
\hline
\end{tabular}

valor médio do segundo componente, significativamente mais alto (Floresta Ripária, $\mathrm{p}=0,001$; e Cordilheira, $\mathrm{p}=0,027)$ do que o do terceiro grupo (Cambarazal). Além disso, foi possível avaliar a variabilidade inter e intrafitofisionomias nesse conjunto de variáveis (Figura 3B). Os resultados indicaram que há alta variabilidade em ambos os componentes do Cambarazal, e a seguinte sequência de magnitude da variabilidade (expressa pelo tamanho das barras de erros), em ordem decrescente, pode ser definida: Cambarazal $>$ Espinheiral $>$ Floresta Ripária $>$ Cordilheira.

Considerando ainda o mesmo conjunto de dados da vazante de 2008, quando se avaliou a participação do $\mathrm{pH}$, do teor de silte e do teor de argila no teor de matéria orgânica do solo, foi possível identificar, por meio de regressão múltipla, que esses fatores ( $\mathrm{pH}$, teor de argila e de silte) foram significativos $(F=74,95 ; p<0,001)$ e explicaram cerca de $80 \%$ dos dados observados $\left(\mathrm{R}^{2}\right.$ ajustado $\left.=0,80\right)$ nas fitofisionomias estudadas (Tabela 2).
O estoque de COS variou dentro de cada fitofisionomia ao longo do ciclo hidrológico e dentro de cada ciclo, segundo a fitofisionomia (Figura 4). Verificou-se que, no que concerne às variações sazonais do estoque de carbono nos solos, o que há de comum nas fitofisionomias, à exceção da cordilheira. Os períodos de enchente e de vazante de 2008 foram aqueles em que se verificaram os maiores e os menores valores da variável, respectivamente. Já na Cordilheira o maior estoque de COS foi observado na cheia.

Em todos os períodos sazonais (Figura 4), os estoques de carbono no solo encontrados no grupo de maior fertilidade, Cambarazal e Espinheiral, foram superiores aos observados no de menor fertilidade, Floresta Ripária e Cordilheira.

\section{DISCUSSÃO}

A determinação dos três grupos distintos entre as fitofisionomias observadas neste trabalho, através

Revista Árvore, Viçosa-MG, v.39, n.2, p.325-336, 2015 
Tabela 2 - Valor dos parâmetros de regressão múltipla para explicar o teor de matéria orgânica do solo em função do pH e dos teores de silte e de argila.

Table 2 - Value of the parameters of multiple regression to explain the content of soil organic matter according to the pH and the silt and clay.

\begin{tabular}{|c|c|c|c|c|}
\hline Variável & Coeficiente de regressão & Erro-padrão & Valor da estatística $\mathrm{t}$ & Nível de probabilidade \\
\hline Intercepto & 130,9727 & 47,6097 & 2,751 & 0,0069 \\
\hline $\mathrm{pH}(\mathrm{x} 1)$ & $-26,0729$ & 9,8761 & $-2,640$ & 0,0094 \\
\hline Teor de silte $(\times 2)$ & $-1,1393$ & 0,3437 & $-3,315$ & 0,0012 \\
\hline Teor de argila (x3) & $-1,2449$ & 0,2338 & $-5,325$ & 0,0000 \\
\hline $\mathrm{x} 1, \mathrm{x} 2$ & 0,2675 & 0,0705 & 3,796 & 0,0002 \\
\hline$x 2, x 3$ & 0,2596 & 0,0501 & 5,177 & 0,0000 \\
\hline $\mathrm{x} 1, \mathrm{x} 3$ & 0,0050 & 0,0009 & 5,327 & 0,0000 \\
\hline$x 1, x 2, x 3$ & $-0,0011$ & 0,0002 & $-5,398$ & 0,0000 \\
\hline
\end{tabular}

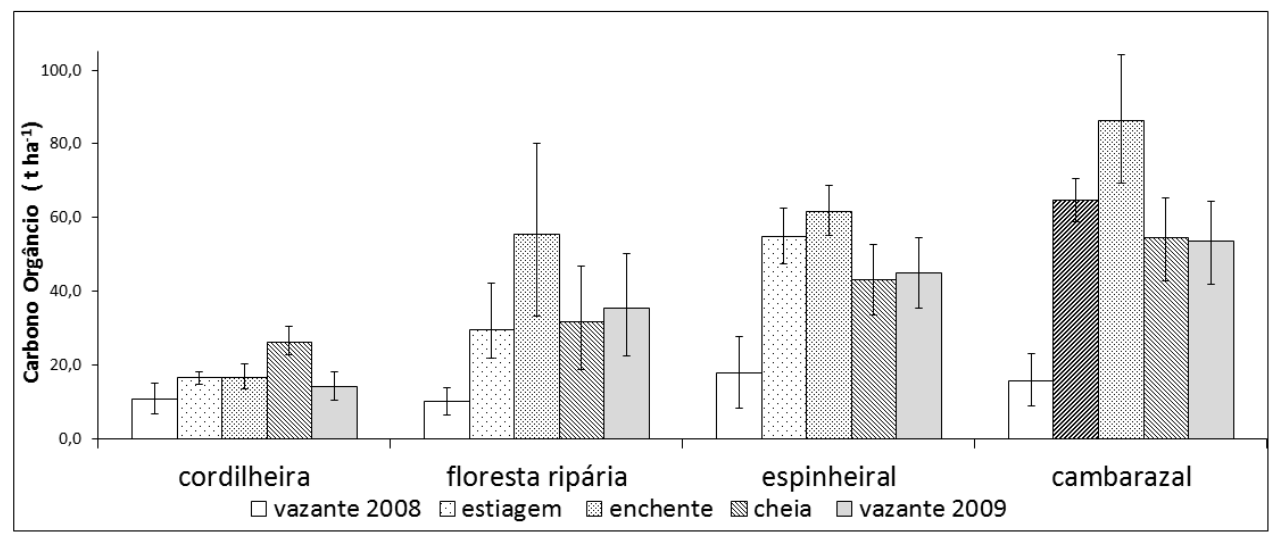

Figura 4 - Estoque de carbono na profundidade de 0 - $20 \mathrm{~cm}$ (médias e intervalos de confiança a 95\%) de cada fitofisionomia ao longo do ciclo hidrológico. As médias e os intervalos de confiança foram calculados empregando a técnica de "bootstrap", com 1.000 reamostragens aleatórias com reposição.

Figure 4-Carbon stock in the 0 - $20 \mathrm{~cm}$ depth (means and confidence intervals at 95\%) of each vegetation type along the hydrological cycle. The means and confidence intervals were calculated using the technique of "bootstrap" with 1,000 random resampling with replacement.

da PCA, foi possível, segundo Carvalho et al. (2011) e Valladares et al. (2008), devido à consistência entre as variáveis envolvidas.

As variáveis observadas neste trabalho corroboram a afirmação de Van Noordwijk et al. (1997) de que, em regiões alagadas, o teor de carbono no solo aumenta em áreas com baixo $\mathrm{pH}$ e elevados teores de silte e argila, entre outros fatores. Isso apesar de as análises feitas por esses pesquisadores terem sido realizadas com a M.O. e não com o teor de carbono. Entretanto, sabe-se que há estreita relação entre ambas as variáveis (PRIBYL, 2010), o que torna igualmente válida tal afirmação.

Esses resultados sugerem que há contribuição de fatores sazonais sobre o estoque de carbono no solo. Entretanto, não foi possível identificar quais fatores seriam esses, porque não foram realizadas medidas de produção, qualidade e acúmulo de serrapilheira e nem de quantificação da contribuição da matéria orgânica carregada pela água superficial de inundação. Essas informações ainda estão ausentes no Pantanal e são importantes para determinar o potencial das fitofisionomias em modificar as características químicas dos solos (VOURLITIS et al., 2011).

Há informações de que os solos pantaneiros guardam estreita relação com a natureza dos sedimentos depositados, o que, por sua vez, é consequência da natureza do material-fonte e dos processos ou formas de deposição/sedimentação (COUTO; OLIVEIRA, 2011; CORINGA et al., 2012). No entanto, há pesquisadores

Revista Árvore, Viçosa-MG, v.39, n.2, p.325-336, 2015 
que sustentam que o estoque de carbono é mais dependente do tipo de fitofisionomia do que do ciclo hidrológico, especialmente quando se comparam ambientes com distintas capacidades de produção de serapilheira (LIMA et al., 2011).

O teor de COS é controlado pelo ambiente e pelos processos pedogenéticos. A passagem de uma fase anóxica no período de cheia para o de seca extrema na estiagem é também fator que governa a dinâmica do estoque de carbono no solo (SAHRAWAT; SPARKS, 2003). Independentemente da natureza de origem da M.O., os valores do estoque de carbono observados nos períodos sazonais da hidrologia característicos da região são devidos também ao fato de que a condição de anaerobiose favorece o acúmulo de matéria orgânica nos solos, porque nessa situação as taxas de decomposição são reduzidas por conta da diminuição da atividade microbiana (ALEWELL et al., 2008).

Bem no início da vazante, por inércia, os valores ainda permanecem elevados, porém passam a diminuir rapidamente a partir do momento em que a pressão parcial de $\mathrm{O}_{2}$ aumenta. Todavia, ainda existe suficiente umidade no solo para promover grande atividade dos microrganismos responsáveis pela decomposição. O subsequente incremento no período de estiagem pode estar associado ao que McClain et al. (2003) denominaram "Hot moments", que são períodos curtos de tempo que apresentam, desproporcionalmente, reações biogeoquímicas com taxas muito elevadas, em comparação com a série temporal do fenômeno. Há de se considerar também que o acúmulo de matéria orgânica não depende apenas da produtividade das plantas, mas também da taxa de decomposição das plantas mortas e do material senescente (COLLINS; KUEHL, 2001), que se acentua principalmente na estiagem pantaneira.

Apesar das diferenças edafoclimáticas próprias dos diferentes ecossistemas terrestres, o padrão sazonal da variação na quantidade de carbono no solo observado neste trabalho também foi identificado por Cerón-Breton et al. (2011).

Com relação ao período de vazante, o comportamento do estoque de carbono no solo observado em 2008 diferiu daquele ocorrido em 2009, na maioria das fitofisionomias. Condição essa que pode estar relacionada à contribuição microbiológica que atua nos solos das diferentes fitofisionomias deste estudo. Taques (2012) avaliou que a contribuição da microbiota edáfica na dinâmica do carbono em solos do Pantanal indica que há diminuição na taxa de turnover do carbono, via mineralização microbiana, durante a mudança de ambiente terrestre para aquático, causada pela inundação. Contudo, é o espaço de tempo dentro desse período sazonal, em que as condições ambientais permanecem favoráveis para a atividade microbiana, que vai determinar em que momento, entre o período de cheia e o de vazante, o estoque de carbono do solo passa a diminuir ou aumentar, dependendo da fitofisionomia. É sabido que há explosão da atividade microbiana assim que as condições edafoclimáticas são favoráveis - o efeito Birch -, o que consequentemente leva à redução significativa do carbono no solo (JARVIS et al., 2007); porém, à medida que as condições edafoclimáticas para a explosão de atividade microbiana diminuem, igualmente há redução significativa do processo de decomposição e mineralização da matéria orgânica do solo.

Nogueira et al. (2002) mostraram que mais de 90\% do carbono orgânico da biomassa terrestre num campo inundável no Pantanal Mato-Grossense foi removido pela água e, ou, perdido pela mineralização da matéria orgânica durante a época de seca. Essa condição não foi observada neste trabalho, pois, considerando que o processamento do carbono em solos com caraterísticas redoxomórficas, como é o caso do solo deste estudo, é complexo e envolve uma série de reações que ocorrem em diferentes horizontes do solo ao longo do ciclo hidrológico (KAYRANLI et al., 2010). A liberação dessa matéria orgânica não acontece continuamente, pois é muito mais um pulso de liberação (MCCLAIN et al., 2003).

As fitofisionomias Cambarazal e Espinheiral, por se localizarem em regiões mais baixas, têm maior probabilidade de apresentar incremento no estoque de carbono do solo durante os períodos de enchente e cheia em relação à Floresta Ripária e à Cordilheira. Como a matéria orgânica está suspensa na água, ao adentrar o período da vazante essa matéria acondiciona-se no solo, aumentando, consequentemente, o teor de carbono e o aporte de nutrientes do local. Um estudo sobre o efluxo de $\mathrm{CO}_{2}$ realizado na Fitofisionomia Cordilheira (JOHNSON et al., 2012) revelou que o efluxo de $\mathrm{CO}_{2}$ do solo da Cordilheira se aproximou de $1.390 \mathrm{~g}$ $\mathrm{C} \mathrm{m}^{-2} \mathrm{a}^{-1}$. Esse valor equivalente a $13,9 \mathrm{t} \mathrm{C} \mathrm{ha}^{-1}$, que é quase igual à diferença entre os estoques máximo e mínimo medidos nesse ambiente $\left(13,8 \mathrm{tC} \mathrm{ha}^{-1}\right)$, teve

Revista Árvore, Viçosa-MG, v.39, n.2, p.325-336, 2015 
um máximo de 26,3 tha-1 na cheia e um valor mínimo de $12,5 \mathrm{t} \mathrm{ha}^{-1}$ durante a vazante (valores de estoque referem-se à profundidade de $0 \mathrm{a} 20 \mathrm{~cm}$ ).

\section{CONCLUSÕES}

1. A análise de componentes principais possibilitou separar as fitofisionomias em três grupos distintos, através da caracterização dos dois primeiros PCAs, sendo o primeiro reserva de nutrientes (fases minerais e orgânicas) e o segundo, componente pela acidez do solo.

2. Verificou-se que no Pantanal Mato-Grossense o estoque de carbono do solo está condicionado por fatores sazonais e igualmente dependentes da fitofisionomia local.

3. As fitofisionomias com maior estoque de carbono no solo foram o Cambarazal e o Espinheiral, as quais igualmente foram as que apresentaram maior nível de fertilidade natural.

3. O período de enchente caracteriza-se por ser aquele em que o estoque de carbono é maior em grande parte das fitofisionomias.

4. Em todas as fitofisionomias, o menor estoque de carbono ocorre no período de vazante (2008), após o período de anaerobiose em razão da enchente e antes que o período da seca passe a ser determinante.

\section{AGRADECIMENTOS}

Ao Conselho Nacional de Desenvolvimento Científico e Tecnológico (CNPq), ao Programa de Estudos Ecológicos de Longa Duração (PELD). ao Instituto Nacional de Ciência e Tecnologia em Áreas Úmidas (INAU) e ao Programa Institutos Nacionais de Ciência e Tecnologia (CNPq/MCT), pois esta pesquisa é uma produção científica do Laboratório 2: Interações Aquático-Terrestres e Fluxos de Carbono do INAU.

\section{REFERÊNCIAS}

ALEWELL, C.; PAUL, S.; LISCHEID, G.; STORCK, F.R.; Co-regulation of redox processes in freshwater wetlands as a function of organic matter availability? Science of the Total Environment, v.404, n.2-3, p.335-342, 2008.

BECHTOLD, J.; NAIMAN, R. A quantitative model of soil organic matter accumulation during floodplain primary succession. Ecosystems, v.12, n.8, p.1352-1368, 2009.

BIRCH, H.F. Mineralisation of plant nitrogen following alternate wet and dry conditions. Plant and Soil, v.20, n.1, p.43-49, 1964..

Bittencourt, A. Propriedades químicas de um Planassolo após 12 anos de cultivo sob diferentes sistemas. 1999. $40 \mathrm{f}$. Dissertação (Mestrado em Agronomia) Universidade Federal de Pelotas, Pelotas, 1999.

BRADY, N.C.; WEIL, R.R. The nature and properties of soils. 12.ed. Upper Saddle River: Prentice Hall, 1999.

BRETON-CERON, J,G.; CERON-BRETON, R.M.; RANGEL-MARRON, M.; MURIEL-GARCIA, M.; CORDOVA-QUIROZ,A.V.; ESTRELL-CAHUICH, A. Determination of carbon sequestration rate in soil of a mangrove forest in Campeche, Maxico, titulo do artigo. International Journal of Energy and Environment, v.5, n.3, p.328336, 2011.

CHRISTIE, D. Resampling with Excel. Teaching Statistics, v.26, n.1, p.9-14, 2004.

COLLINS, M.E.; KUEHL, R.J. Organic matter accumulation and organic soils. In: RICHARDSON, J.L.; VEPRASKAS, M.J. (Ed.). Wetland soils: Genesis, hydrology, landscapes and classification. Boca Raton: CRC Press, 2001. p.137-162.

CORINGA, E.A.O.; COUTO, E.G.; PEREZ, X.L.O.; TORRADO, P.V. Atributos de solos hidromórficos no Pantanal Norte Matogrossense. Acta Amazonica, v.42, p.19-28, 2012.

COUTO, E.G.; OLIVEIRA, V.A. The Soil diversity of the Pantanal In: JUNK, W. .;DA SILVA, C.J., demais editores (Ed.). The Pantanal of Mato Grosso: Ecology, biodiversity and sustainable management of a large neotropical seasonall wetland. Sofia: Pensoft, 2011. p.71-102.

CRASWELL, E.T.; LEFROY, R.D.B. The role and function of organic matter in tropical soils.

Nutrient Cycling in Agroecosystems, v.61, n.1-2, p.7-18, 2001. 
DILLON, W.R.; GOLLDSTEIN, M. Multivariate analysis: methods and applications. New York: John Wiley \& Sons, 1984.

EAGLE, A.J.; OLANDER, L.P. Greenhouse gas mitigation with agricultural land management activities in the United States - A Side-by-Side comparison of biophysical potential. In:

DONALD, L.S. (Ed.). Advances in Agronomy. New York: Academic Press, 2012. v.115. p. $79-179$.

GUO, J.; ZHANG, W.; ZHANG, M.; ZHANG, L.; BIAN, X. Will elevated $\mathrm{CO}_{2}$ enhance mineral bioavailability in wetland ecosystems? Evidence from a rice ecosystem. Plant and Soil, p. 1$13,2012$.

HAIR JR., J. F.; ANDERSON, R. E.; TATHAM, R. L.; BLACK, W. C. Análise multivariada de dados. 5.ed. Porto Alegre: Bookman, 2005.

HASENACK, H.; CORDEIRO, J.L.P.; HOFMANN, G.S. O clima da RPPN SESC Pantanal. Relatório técnico. Porto Alegre: UFRGS - IB Centro de Ecologia - Laboratório de Geoprocessamento, 2003. 19 p.

HOLDEN, J. Peatland hydrology and carbon release: why small-scale process matters.

Philosophical Transactions of the Royal Society A: Mathematical, Physical and Engineering Sciences, v. 363 , n. 1837 , p. 2891-2913.

INSTITUTO BRASILEIRO DE GEOGRAFIA E ESTATÍSTICA - IBGE. Manual técnico da vegetação brasileira. Rio de Janeiro: 1992. $92 \mathrm{p}$.

JARVIS, P.; REY, A.; PETSIKOS, C.; WINGATE, L.; RAYMENT, M.; PEREIRA J.; BANZA, J; DAVID, J.; MIGLIETTA, F.; BORGHETTI, M; MANCA G.; VALENTINI, R. Drying and wetting of Mediterranean soils stimulates decomposition and carbon dioxide emission: the "Birch effect". Tree Physiology, v.27, n.7, p.929-940, 2007.

MARK, S.J.; COUTO, E.G; PINTO JR., O.B; MILESI, J.; AMORIM, R.S.S.; MESSIAS, I.A.M.; BIUDES, M.S. Soil $\mathrm{CO}_{2}$ dynamics in a Tree Island Soil of the Pantanal: the role of soil water potential. Plos One, v.8, n.6, p. e64874, 2013.
JOHNSTON, A.E.; POULTON, P.R.; COLEMAN, K. Soil organic matter: Its importance in sustainable agriculture and carbon dioxide fluxes. In: DONALD, L.S. (Ed.). Advances in Agronomy. New York: Academic Press, 2009. v.101.p.1-57.

JOLLIFFE, I.T. Principal component analysis. 2.ed. New York: Springer, 2002. 487p.

JUNK, W.J.; NUNES DA CUNHA, C.; WANTZEN, K.M.; PETERMANN, P.; STRUSSMANN, C.; MARQUES, M.I.; ADIS, J. Biodiversity and its conservation in the Pantanal of Mato Grosso, Brazil.

Aquatic Sciences, v.68, p.278-309, 2006.

KAYRANLI, B.; SCHOLZ, M.; MUSTAFA, A.; HEDMARK, $\AA$. Carbon storage and fluxes within Freshwater Wetlands: a critical review. Wetlands, v.30, n.1, p.111-124, 2010.

LIMA, S. S.; LEITE, L. F. C.; OLIVEIRA, F. C.; COSTA, D. B. Atributos químicos e estoques de carbono e nitrogênio em argissolo vermelhoamarelo sob sistemas agroflorestais e agricultura de corte e queima no norte do Piauí. Revista Árvore, v.35, n.1, p.51-60, 2011.

LONG, G.L.; WINEFORDNER, J.D. Limit of detection. A closer look at the IUPAC definition. Analytical Chemistry, v. 55, n.7, p.712A-724A, 1983.

MCCLAIN, M. E.; BOYER, E. W.; DENT, C. L.; GERGEL, S. E.; GRIMM, N. B.; GROFFMAN, P. M.; HART, S. C.; HARVEY, J. W.; JOHNSTON, C. A.; MAYORGA, E.; MCDOWELL, W. H.; PINAY, G. Biogeochemical hot spots and hot moments at the interface of terrestrial and aquatic ecosystems. Ecosystems, v.6, n.4, p.301-312, 2003.

MITSCH, W.J.; GOSSELINK, J.G. The value of wetlands: importance of scale and landscape setting. Ecological Economics, v. 35 , n. 1 , p. $25-33,2000$

NEUE, H. U. Organic matter dynamics in wetland soils. In: Wetland Soils: Characterization, Classification, and Utilization. Proceedings of a workshop held 26 March to 5 April 1984. Los Baños: International Rice Research Intitute, 1985. p.109-122.

Revista Árvore, Viçosa-MG, v.39, n.2, p.325-336, 2015 
NOGUEIRA, F.; COUTO, E.G.; BERNARDI, C.J. Geostatistics as tool to improve sampling and statistical analysis in wetlands: a case study on dynamics of organic matter distribution in the Pantanal of MatoGrosso, Brazil. Brazilian Journal of Biology, v.62, n.4B, p.861-870, 2002.

NUNES DA CUNHA, C.; JUNK, W.J. Distribution of wood plant communities along the flood gradient in the Pantanal of Poconé, Mato Grosso, Brazil. International Journal of Ecology and Environmental, v.27, n.1, p.63-70, 2001.

PRIBYL, D.W. A critical review of the conventional SOC to SOM conversion factor. Geoderma, v.156, n.3-4, p.75-83, 2010.

REIS, E. Estatística multivariada aplicada. 2.ed. Lisboa: Silabo, 2001.

RODRIGUES, L.A.; CARVALHO, D. A.; OLIVEIRA FILHO, A. T.; CURI, N. Efeitos de solos e topografia sobre a distribuição de espécies arbóreas em um fragmento de floresta estacional semidecidual, em Luminárias, MG. Revista Árvore, v. 31, n.1, p.25-35, 2007.

SAHRAWAT, K. L.; SPARKS, D. L. Organic matter accumulation $\mathrm{n}$ submerged soils. Advances in Agronomy, v.81, p.169-201, 2004.

SANTOS, R.D.; LEMOS, R.C.; SANTOS, H.G.; KER, J.; ANJOS, L.H.C. Manual de descrição e coleta de solo no campo. 5a edição. 5.ed. Viçosa, MG: Sociedade Brasileira de Ciência do Solo/Centro Nacional de Pesquisa em Solos, 2005. 83p.
SILVA, M.P.; MAURO, R.; MOURÃO, G.; COUTINHO, M. Distribuição e quantificação de classes de vegetação do Pantanal através de levantamento aéreo. Revista Brasileira de Botânica, v.23, n.2, p.143-152, 2000.

SMITH, P.; FANG, C.M.; DAWSON, J.J.C.; MONCRIEFF, J. Impact of global warming on soil organic carbon. In: DONALD, L. S. (Ed.). Advances in Agronomy. New

York:Academic Press, 2008. v.97.p.1-43. ISBN 0065-2113.

SPSS. IBM SPSS Statistics 19 Brief

Guide. SPSS, 2010.171p.

TAQUES, L.P. Dinâmica do carbono no solo frente à mudança do ambiente terrestre - aquático no Pantanal Matogrossense. 2012. 50f. Dissertação (Mestrado em Ecologia e Conservação da Biodiversidade) - Universidade Federal do Mato Grosso, Cuiabá, 2012.

NOORDWIJK, M.V.; CERRI, C.C.; WOOMER, P.L.; NUCROHO, K.; BERNOUX, M. Soil carbon dynamics in the humid tropical forest zone. Geoderma, v.79, n.1-4, p.187-225, 1997.

VOURLITIS, G.L.; LOBO, F.A.; BIUDES, M.S.; ORTÍZ, C.E.R.; NOGUEIRA, J.S. Spatial variations in soil chemistry and organic matter content across a invasion front in the Brazilian Pantanal. Soil Science Society of America Proceedings, v.75, n.4, p.1554-1561, 2011 